\title{
Enhanced Strain-Dependent Electrical Resistance of Polyurethane Composites with Embedded Oxidized Multiwalled Carbon Nanotube Networks
}

\author{
R. Benlikaya, ${ }^{1,2}$ P. Slobodian, ${ }^{1,3}$ and P. Riha $^{4}$ \\ ${ }^{1}$ Centre of Polymer Systems, University Institute, Tomas Bata University, Nad Ovcirnou 3685, 76001 Zlin, Czech Republic \\ ${ }^{2}$ Balikesir University, Faculty of Necatibey Education, Department of Secondary Science and Mathematics Education, 10100 Balikesir, \\ Turkey \\ ${ }^{3}$ Tomas Bata University, Faculty of Technology, Polymer Centre, TGM 275, 76001 Zlin, Czech Republic \\ ${ }^{4}$ Institute of Hydrodynamics, Academy of Sciences, Pod Patankou 30/5, 16612 Prague 6, Czech Republic
}

Correspondence should be addressed to P. Slobodian; slobodian@ft.utb.cz

Received 15 March 2013; Accepted 18 August 2013

Academic Editor: Sulin Zhang

Copyright (C) 2013 R. Benlikaya et al. This is an open access article distributed under the Creative Commons Attribution License, which permits unrestricted use, distribution, and reproduction in any medium, provided the original work is properly cited.

\begin{abstract}
The effect of different chemical oxidation of multiwalled carbon nanotubes with $\mathrm{H}_{2} \mathrm{O}_{2}, \mathrm{HNO}_{3}$, and $\mathrm{KMnO}_{4}$ on the change of electrical resistance of polyurethane composites with embedded oxidized nanotube networks subjected to elongation and bending has been studied. The testing has shown about twenty-fold increase in the electrical resistance for the composite prepared from $\mathrm{KMnO}_{4}$ oxidized nanotubes in comparison to the composites prepared from the pristine and other oxidized nanotubes. The evaluated sensitivity of $\mathrm{KMnO}_{4}$ treated composite in terms of the gauge factor increases with strain to nearly 175 at the strain $11 \%$. This is a substantial increase, which ranks the composite prepared from $\mathrm{KMnO}_{4}$ oxidized nanotubes among materials as strain gauges with the highest electromechanical sensitivity. The observed differences in electromechanical properties of the composites are discussed on basis of their structure which is examined by the measurements of Fourier transform infrared spectroscopy, X-ray photoelectron spectroscopy, and scanning electron microscope. The possible practical use of the composites is demonstrated by monitoring of elbow joint flexion during two different physical exercises.
\end{abstract}

\section{Introduction}

In recent years there has been an increase in the studies on the composites composed of thermoplastic polyurethane (TPU) with carbon nanomaterials such as multiwalled carbon nanotubes (MWCNTs) [1], carbon nanofibers [2], and single- or multi-walled carbon nanotube networks (SWCNT-N, MWCNT-N), or buckypaper [3-5]. Thermoplastic polyurethane elastomers exhibit useful properties as abrasion resistance. However, since these polymers are not chemically cross-linked, they exhibit lower recoverability following elongation than cross-linked elastomers [6].

The carbon nanotube networks (CNT-Ns) can proportionally transfer their unique properties into composites and bring about substantial improvements in their properties such as the strength, the electrical and thermal conductivity, the electromagnetic interference shielding compared to the polymer composites with CNT particulate filler [7]. Using CNT-Ns as a strain sensor has also potential advantage since the networks have high sensitivity to local distributions of stress and strain as well as the ability to sense stresses and strains in different directions in the host materials [8]. In this respect the strain sensitivities of epoxy [8], poly (methylmethacrylate) [9], and thermoplastic polyurethane composites [5] with MWCNT-Ns or SWCNT-Ns have already been studied. Moreover, there are also some studies on the effect of the functionalization of CNT-Ns with plasma $[10,11]$ as well as the carboxylic acid and amine groups [5] on 
the electrical properties [5, 11], thermal properties [5], and mechanical properties $[10,11]$ of polyurethane [5], polyimide [10] and polycarbonate [11] composites with CNT-Ns. Lima and coworkers stated that the range of property design possibilities of the elastomeric polyurethane composite is possible by tailoring the functional group content and the carbon nanotube load [5]. Nevertheless, according to our knowledge, no study related to examination of the effect of functionalized MWCNT-Ns with various oxidants on the strain sensitivity of TPU/MWCNT-N composites has been published yet. There are some studies showing various properties of CNT-polymer bulk composites to be affected by various oxidation conditions of nanotubes [12]. Rein and coworkers investigated the coupling between the electrical and mechanical properties of the CNT-N films made of SWCNTs and MWCNTs when embedded in several types of epoxies and observed different electromechanical responses [8]. However, this coupling is not affected only by the nanotube type but also by the interaction of the polymer matrix and CNT-N films [13].

In this study, the thermoplastic polyester-based polyurethane composite with various MWCNT-Ns, oxidized by three agents as $\mathrm{H}_{2} \mathrm{O}_{2}, \mathrm{HNO}_{3}$, and $\mathrm{KMnO}_{4}$, is investigated in terms of their electrical resistance change during elongation and bending. The observed differences in electromechanical properties of TPU composites are discussed on basis of their structure which is examined by the Fourier transform infrared spectroscopy (FTIR), the X-ray photoelectron spectroscopy (XPS), and the scanning electron microscope (SEM).

\section{Experimental}

2.1. Materials. Purified MWCNTs produced by the chemical vapor deposition of acetylene were supplied by Sun Nanotech Co. Ltd., China, and characterized in our previous studies [4, $7,14]$. The thermoplastic polyurethane elastomer Desmopan DP 2590A was supplied by Bayer Material Science.

$\mathrm{KMnO}_{4}$ oxidation: the oxidized MWCNTs were prepared in a glass reactor with a reflux condenser filled with $250 \mathrm{~mL}$ of $0.5 \mathrm{M} \mathrm{H}_{2} \mathrm{SO}_{4}$, into which $5 \mathrm{~g}$ of $\mathrm{KMnO}_{4}$ as oxidizing agent and $2 \mathrm{~g}$ of MWCNTs were added. The dispersion was sonicated at $85^{\circ} \mathrm{C}$ for 15 hours using thermostatic ultrasonic bath (Bandelin Electronic DT 103H). The dispersion was filtered, and then MWCNTs were washed with concentrated $\mathrm{HCl}$ to remove $\mathrm{MnO}_{2}$ and then washed with water until $\mathrm{pH}=$ 7.

$\mathrm{HNO}_{3}$ oxidation: $2 \mathrm{~g}$ of MWCNTs was added to $250 \mathrm{~mL}$ of $\mathrm{HNO}_{3}$ (concentrated) and heated at $140^{\circ} \mathrm{C}$ for 2 hours. After that the dispersion was cooled and filtered. The sediment was washed by deionized water and dried at $40^{\circ} \mathrm{C}$ for 24 hours.

$\mathrm{H}_{2} \mathrm{O}_{2}$ oxidation: $1.5 \mathrm{~g}$ of MWCNTs was added to $30 \mathrm{~mL}$ of $\mathrm{H}_{2} \mathrm{O}_{2}$ and simultaneously stirred at $50 \mathrm{rpm}$ (Heidolph, type MR HEI-standard) and heated for 72 hours at $65^{\circ} \mathrm{C}$. Each day $5 \mathrm{~mL}$ of fresh hydrogen peroxide was added to original dispersion to refresh the activity of $\mathrm{H}_{2} \mathrm{O}_{2}$. In the end the dispersion was filtered and rinsed by water. The resulting product was dried at $40^{\circ} \mathrm{C}$ for 24 hours.
For making an entangled MWCNT network, a porous polyurethane membrane and a vacuum filtration method were used. The homogenized dispersion of MWCNTs was vacuum filtered through the membrane. The formed networked layer had thickness about $35 \mu \mathrm{m}$. The partial infiltration of MWCNTs into the filter pores creates an effective interlocking of MWCNT network layer with TPU filter which even strengthens when the porous filter is transformed into the polymeric film in the course of compression molding at $175^{\circ} \mathrm{C}$. The detailed preparation of TPU membrane, MWCNT networks, and the composite was described in our previous paper [4].

The network of pristine MWCNTs is denoted below as MWCNT-N, and the ones of oxidized MWCNTs are denoted as MWCNT-N $\left(\mathrm{KMnO}_{4}\right)$, MWCNT-N $\left(\mathrm{HNO}_{3}\right)$, MWCNT$\mathrm{N}\left(\mathrm{H}_{2} \mathrm{O}_{2}\right)$. The composites of the size $10 \times 30 \mathrm{~mm}$ were melt welded onto the surface of TPU tensile test specimen (dog-bone shaped) for extension and bending tests [4]. The shape and dimension are chosen according to standard EN ISO 3167. The resulting structure of prepared samples sensitive to deformation consists of three-layers structure. There is TPU specimen, TPU/MWCNT composite layer created from melted TPU filtering membrane with interfiltrated nanotubes, and pure MWCNT network originated on filter during filtration.

2.2. Characterization of MWCNT-N and TPU/MWCNT-N Composite. TEM of CNT material was performed using microscope JEOL JEM 2010 at the accelerating voltage of $160 \mathrm{kV}$. The sample for TEM was fabricated on 300 mesh copper grid with a carbon film (SPI, USA) from MWCNT dispersion in acetone prepared by ultrasonication, which was deposited on the grid and dried.

The structure of MWCNT-Ns on the composites was analyzed by scanning electron microscope (SEM) Vega LMU, produced by Tescan Ltd. The samples were deposited on carbon targets and covered with a thin Au/Pd layer. For the observations the regime of secondary electron was chosen.

XPS signals measured from MWCNT-Ns were recorded using a Thermo Scientific K-Alpha XPS system (Thermo Fisher Scientific, UK) equipped with a microfocused, monochromatic $\mathrm{Al} \mathrm{K} \alpha \mathrm{X}$-ray source $(1486.6 \mathrm{eV})$. An X-ray beam of $400 \mu \mathrm{m}$ size was used at $6 \mathrm{~mA} \times 12 \mathrm{kV}$. The spectra were acquired in the constant analyzer energy mode with pass energy of $200 \mathrm{eV}$ for the survey. Narrow regions were collected using the snapshot acquisition mode $(150 \mathrm{eV}$ pass energy) enabling rapid collection of data ( $5 \mathrm{~s}$ per region). The narrow region data was postprocessed using Jansson's algorithm to remove the analyser point spread function which resulted in improved resolution of the spectra for peak deconvolution [15].

The resistance change of the composites was monitored by a two-point technique under different tensile loading. The time-dependent resistance change was measured by means of the Vernier LabQuest Interface System connected to the Differential Voltage Probe and the Wheatstone bridge with sampling frequency $200 \mathrm{~Hz}$.

Fourier transform infrared (FTIR) analyses of the MWCNT-Ns and the composite samples were performed 
TABLE 1: Summary of FTIR measurements for the pristine and oxidized MWCNT-Ns.

\begin{tabular}{|c|c|c|c|c|}
\hline \multirow{2}{*}{ Possible assignments } & \multicolumn{4}{|c|}{ Wavenumber $\left(\mathrm{cm}^{-1}\right)$} \\
\hline & MWCNT-N & $\begin{array}{c}\text { MWCNT-N } \\
\left(\mathrm{H}_{2} \mathrm{O}_{2}\right)\end{array}$ & $\begin{array}{c}\text { MWCNT-N } \\
\left(\mathrm{HNO}_{3}\right)\end{array}$ & $\begin{array}{c}\text { MWCNT-N } \\
\left(\mathrm{KMnO}_{4}\right)\end{array}$ \\
\hline $\mathrm{OH}$ stretch & 3435 & 3459 & 3428 & 3427 \\
\hline $\mathrm{C}-\mathrm{H}$ stretch $\left(\mathrm{CH}_{2}, \mathrm{CH}_{3}\right)$ & 2908,2840 & 2977,2888 & 2980,2880 & 2978,2888 \\
\hline $\mathrm{C}=\mathrm{O}$ stretch (carboxyl or ketone) & 1705 & 1718 & 1726 & 1710 \\
\hline $\begin{array}{l}\text { Intermediate oxidized } \\
\text { products-quinone groups }\end{array}$ & 1652 & 1637 & 1661,1635 & 1641 \\
\hline $\mathrm{C}=\mathrm{C}$ stretch & 1559 & 1565 & 1580 & 1569 \\
\hline $\mathrm{CH}_{2} / \mathrm{CH}_{3}$ bending & 1460 & 1443 & 1437 & 1440 \\
\hline $\begin{array}{l}\text { Skeletal C-C tangential motions } \\
+\mathrm{C}-\mathrm{O} \text { stretch }\end{array}$ & 1222 & 1219,1167 & 1184 & 1190 \\
\hline $\mathrm{C}-\mathrm{O}$ stretch & 1082 & 1083,1052 & 1084,1049 & 1087,1046 \\
\hline
\end{tabular}

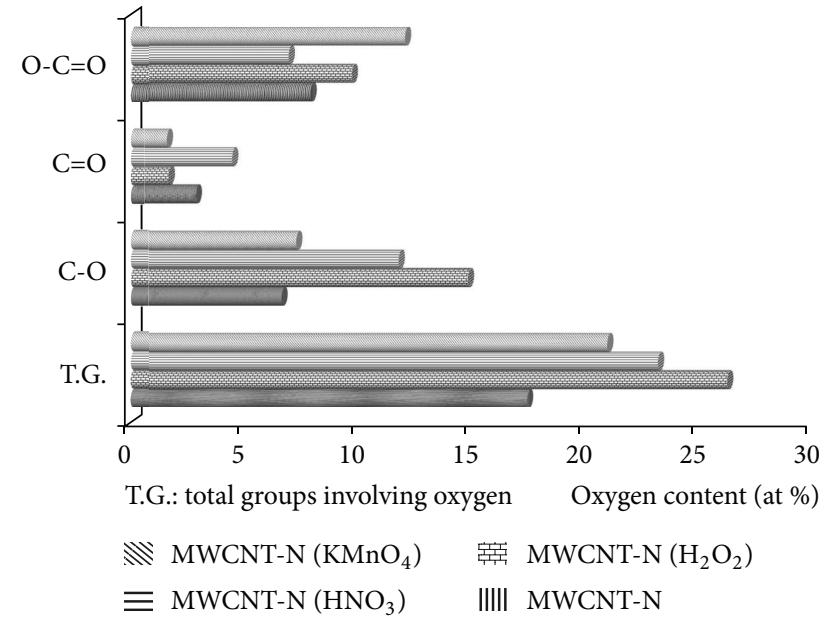

FIGURE 1: Oxygen content (at \%) on MWCNT-Ns oxidized by different reagents.

on FT-IR spektrometr Nicolet 6700. Transmission accessory was used for the MWCNT-Ns samples in powder form prepared by KBr. FTIR-attenuated total reflectance (ATR) spectra of the interface of TPU/MWCNT composite layer where upper network made of pure CNT was washed out were obtained using Ge plate for ATR and repeated three times. The MWCNT-Ns were used for FTIR-ATR spectra of TPU/MWCNT composites as a background.

\section{Results}

3.1. FTIR Measurements. Table 1 summarizes the frequencies of some functional groups in FTIR spectra of MWCNTNs. The $\mathrm{OH}$ stretch is shifted to lower wavelength for only MWCNT-N $\left(\mathrm{H}_{2} \mathrm{O}_{2}\right)$ which could show the increase in the amount of hydrogen bonded hydroxyl groups. On the other hand, C-H stretching of MWCNTs is shifted to lower wavelength for all oxidation treatments.

A weak $\mathrm{C}=\mathrm{O}$ peak at $1705 \mathrm{~cm}^{-1}$ was observed in pristine MWCNT-N, which shows that there is carbonyl or carboxylic group in its surface. The reason why pristine MWCNT$\mathrm{N}$ has carbonyl and $\mathrm{OH}$ groups could be partial oxidation of the surfaces of MWCNTs during purification by the manufacturer [16]. The higher shift in the carbonyl stretching mode is seen for MWCNT-N $\left(\mathrm{HNO}_{3}\right)$ than for other oxidized MWCNT-Ns. The reason may be a kind of $\mathrm{C}=\mathrm{O}$ group or other groups that interacts with the $\mathrm{C}=\mathrm{O}$ group. Zhang and coworkers found that even if the acid mixture and dilute nitric acid produce carboxylic group on the surface of SWCNTs, the strength vibration peaks are not at the same location due to possible different hydrogen bonding interactions [17]. The results summarized in Table 1 also show that there are probably no anhydride/lactone groups on the surfaces of MWCNTs since these groups are usually observed at around $1750 \mathrm{~cm}^{-1}$ or more high wavenumber [18-20].

The peak assigned to quinone group at $1652 \mathrm{~cm}^{-1}$ in pristine MWCNT-N is usually shifted to higher wavelength in oxidized MWCNT-Ns. Coupling effects (i.e., both of intermolecular and inner molecular hydrogen bonding with hydroxyl groups) also might be responsible for the downshift in the $\mathrm{C}=\mathrm{O}$ stretching mode, besides the production of surface-bound quinone groups with extended conjugation [21].

The up-shift in the $\mathrm{C}=\mathrm{C}$ stretching mode of MWCNT-Ns was observed for all oxidized MWCNT-Ns. The highest shift was observed for MWCNT-N $\left(\mathrm{HNO}_{3}\right)$ compared to other MWCNT-N. This treatment may suggest a change in the structure of the MWCNT-N [17].

The $\mathrm{C}-\mathrm{H}\left(\mathrm{CH}_{2} / \mathrm{CH}_{3}\right)$ bending at $1460 \mathrm{~cm}^{-1}$ and the peak at $1222 \mathrm{~cm}^{-1}$ for MWCNT-N are shifted to higher wavelength for all oxidized MWCNT-Ns. The peak at around $1170 \mathrm{~cm}^{-1}$ assigned to $\mathrm{C}-\mathrm{OH}$ group was also observed on MWCNT after $\mathrm{H}_{2} \mathrm{O}_{2}$ oxidation in another study [22]. A new band around $1050 \mathrm{~cm}^{-1}$ in FTIR spectra of oxidized MWCNT-Ns was also observed, which could be assigned to alcoholic CO stretching vibration [22]. Overall, the observed changes in FTIR spectra of oxidized MWCNT-Ns confirm the efficiency of the oxidizing process and formation of the new oxygencontaining functional groups on the surface of carbon nanotubes. 

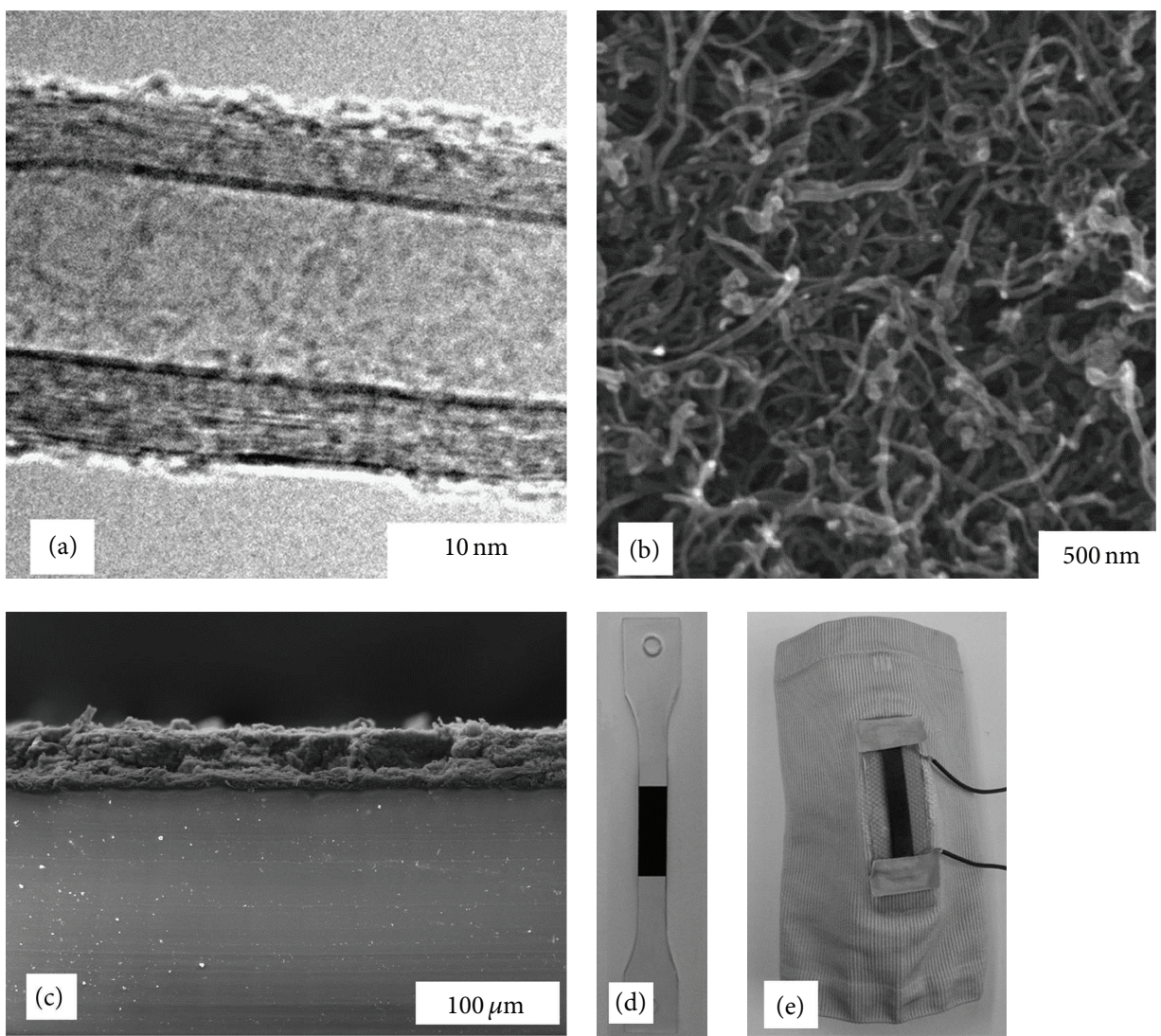

FIGURE 2: (a) TEM micrograph of nanotube structure; (b) SEM micrograph of the surface of entangled MWCNT network; (c) the composite cross-section after the compression molding; (d) photograph of TPU dog-bone shaped specimen; (e) and (a) position of measuring strip of TPU/MWCNT network composite on the elastic elbow bandage.

3.2. XPS Data. The X-ray photoelectron spectroscopy was performed on the MWCNT-N specimens to get information on the functional groups attached onto the nanotube surface. The surface composition (in atomic \%) was determined by considering the integrated peak areas of $\mathrm{Cl}$ and the respective sensitivity factors. The fractional concentration of a particular element $A$ was computed using

$$
\% A=\frac{\left(I_{A} / s_{A}\right)}{\sum\left(I_{n} / s_{n}\right)} \times 100 \%,
$$

where $I_{A}$ denotes the signal intensity of element $A, I_{n}$ integrated peak areas, and $s_{A}$ and $s_{n}$ the Scofield sensitivity factors corrected for the analyzer transmission.

The main binding energy peak $(284.5 \mathrm{eV})$ in XPS spectra of MWCNT-Ns was assigned to the C1s-sp2, while the other ones were assigned to $\mathrm{C}-\mathrm{O}(286.2 \mathrm{eV}), \mathrm{C}=\mathrm{O}(287.1 \mathrm{eV}), \mathrm{O}-$ $\mathrm{C}=\mathrm{O}(288.6-289 \mathrm{eV})$, and $\mathrm{Cls}-\pi-\pi^{*}(291.1-291.5 \mathrm{eV})$. XPS data in Figure 1 show that all MWCNT-Ns have $\mathrm{C}-\mathrm{OH}, \mathrm{C}=\mathrm{O}$, and $\mathrm{O}-\mathrm{C}=\mathrm{O}$ groups on their surface and that MWCNT-N $\left(\mathrm{KMnO}_{4}\right)$, MWCNT-N $\left(\mathrm{HNO}_{3}\right)$, and MWCNT-N $\left(\mathrm{H}_{2} \mathrm{O}_{2}\right)$ have maximum percentage of $\mathrm{O}-\mathrm{C}=\mathrm{O}, \mathrm{C}=\mathrm{O}$, and $\mathrm{C}-\mathrm{OH}$, respectively. FTIR data of these MWCNT-Ns also confirm the presence of these functional groups on the surface. It was also stated in an other study that MWCNTs treated with $\left(\mathrm{NH}_{4}\right)_{2} \mathrm{~S}_{2} \mathrm{O}_{8}, \mathrm{H}_{2} \mathrm{O}_{2}$, and $\mathrm{O}_{3}$ have higher concentrations of carbonyl and hydroxyl functional groups, while more aggressive oxidants (e.g., $\mathrm{HNO}_{3}, \mathrm{KMnO}_{4}$ ) form higher fractional concentrations of carboxyl groups [23]. The acidic potassium permanganate $\left(\mathrm{KMnO}_{4}\right)$ is a strong oxidizing agent and produces more surface acidic groups than nitric acid [24].

The study of Fang and coworkers shows that XPS analysis is a useful and simple method for evaluating the content variation of amorphous carbon in SWNT samples by analyzing the $\mathrm{sp}^{3} / \mathrm{sp}^{2}$ carbon ratio [25]. According to our XPS results of MWCNT-Ns the carbon ratios are 2.50, 2.71, 3.50, and 1.69 for MWCNT-N, MWCNT-N $\left(\mathrm{H}_{2} \mathrm{O}_{2}\right)$, MWCNT-N $\left(\mathrm{HNO}_{3}\right)$, and MWCNT-N $\left(\mathrm{KMnO}_{4}\right)$, respectively. The reason why the total oxygen amount for MWCNT-N $\left(\mathrm{KMnO}_{4}\right)$ is less than that in case of other oxidants could be due to removing away more amount of the oxygen species originated from amorphous carbon structures during the acidified $\mathrm{KMnO}_{4}$ oxidation process.

3.3. From Tubes to Strain Sensing Element. Figures 2(a)-2(e) summarize pathway of CNT application for strain sensing element. Part (a) represents TEM analyses of one individual MWCNT tube which typically consists of about 15-35 rolled layers of graphene. The SEM micrograph of the upper surfaces of MWCNT-N network created from entangled tubes is presented in part (b). Comparing results for each principal 


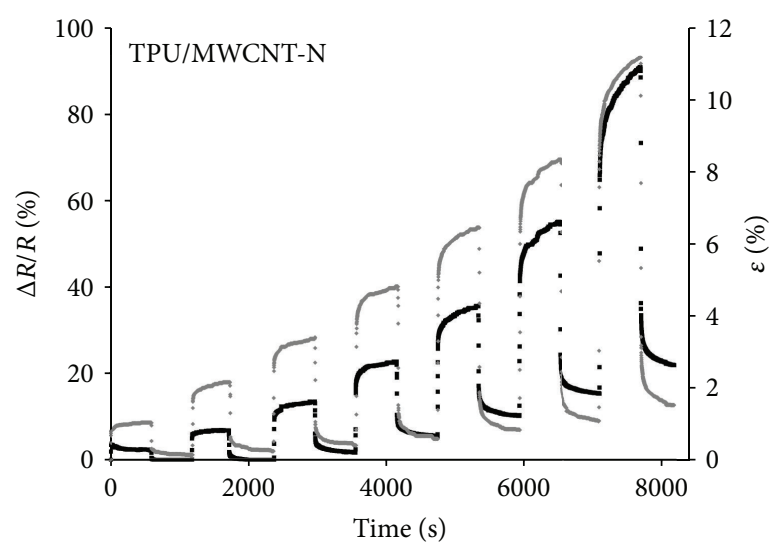

(a) TPU/MWCNT-N

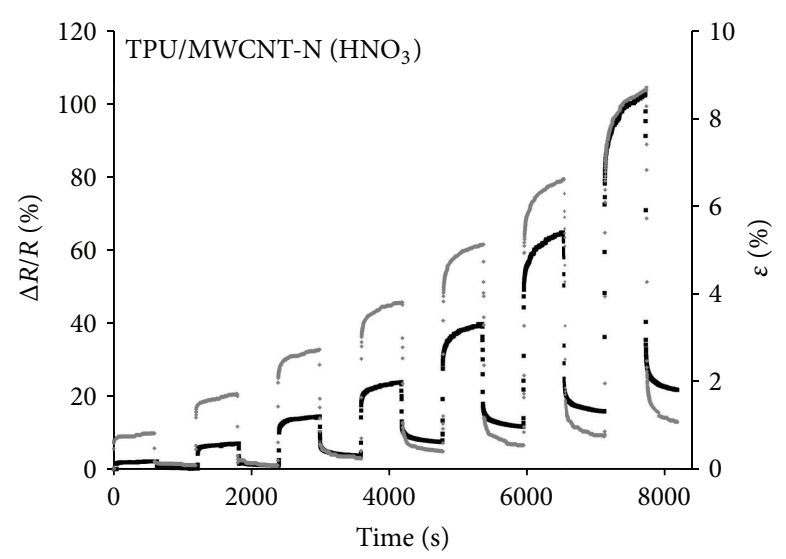

(c) TPU/MWCNT-N $\left(\mathrm{HNO}_{3}\right)$

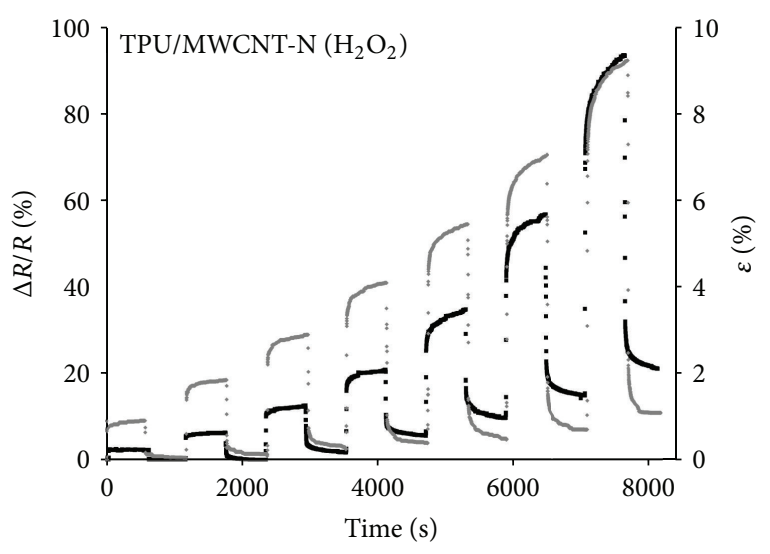

(b) TPU/MWCNT-N $\left(\mathrm{H}_{2} \mathrm{O}_{2}\right)$

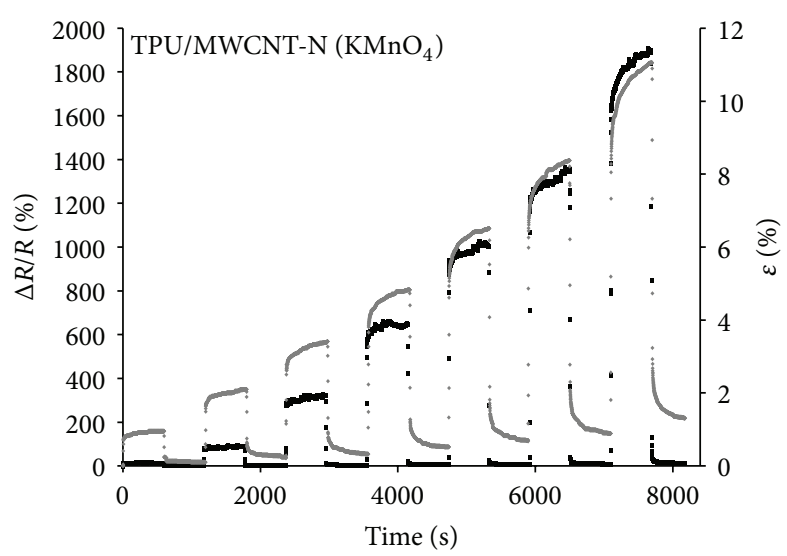

(d) TPU/MWCNT-N $\left(\mathrm{KMnO}_{4}\right)$

FIGURE 3: Response of the relative resistance change and strain of the TPU composites versus the step increase of tensile strength (a) TPU/MWCNT-N, (b) TPU/MWCNT-N $\left(\mathrm{H}_{2} \mathrm{O}_{2}\right)$, (c) TPU/MWCNT-N $\left(\mathrm{HNO}_{3}\right)$, and (d) TPU/MWCNT-N $\left(\mathrm{KMnO}_{4}\right)$.

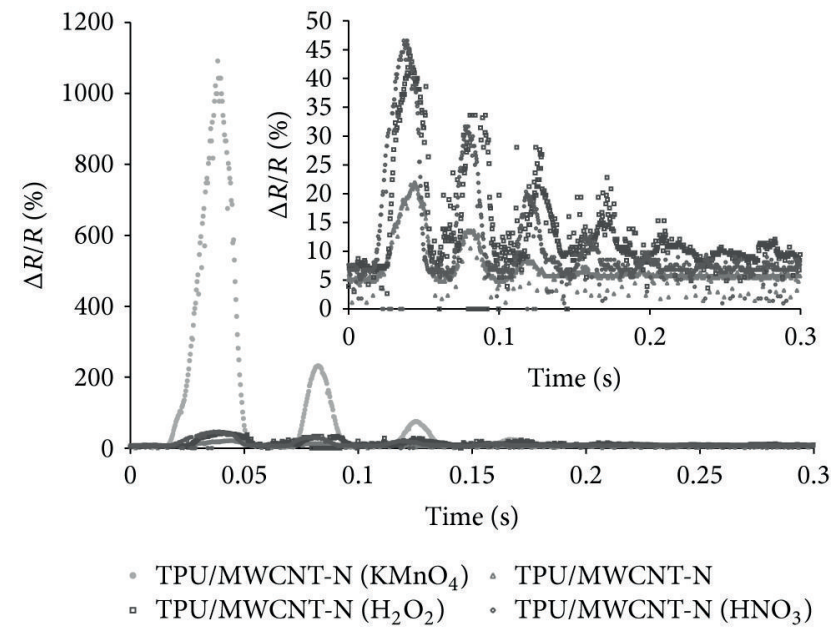

FIgURE 4: Time-dependent response of the strips of the TPU composites anchored tightly on one side to the initial deflection of free ending.

material it was found that the structures of networks slightly differ from each to other. MWCNT-N $\left(\mathrm{KMnO}_{4}\right)$ seems to

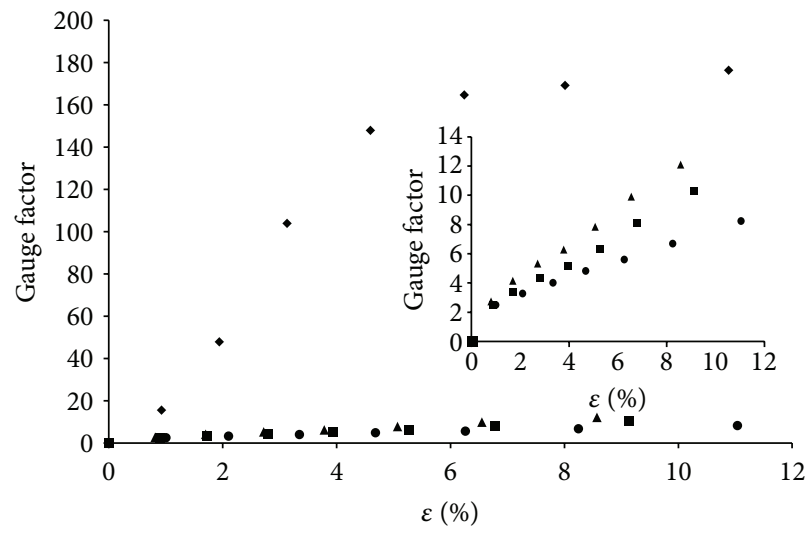

$\begin{array}{ll}\text { - TPU/MWCNT-N }\left(\mathrm{KMnO}_{4}\right) & \text { - TPU/MWCNT-N }\left(\mathrm{H}_{2} \mathrm{O}_{2}\right) \\ \text { - TPU/MWCNT-N }\left(\mathrm{HNO}_{3}\right) & \text { - TPU/MWCNT-N }\end{array}$

FIgURE 5: Gauge factors of the TPU composites.

have smoother surface than others with more densely packed nanotubes and smaller diameters of pores. On the other hand, the most porous structure belongs to MWCNT-N $\left(\mathrm{HNO}_{3}\right)$. The composite cross-section after the compression molding is 
<smiles>COC(=O)CCCCC(=O)OCCCCOC(=O)OCCCCOC(=O)Nc1ccc(Cc2ccc(N(I)C(=O)OCCCCOC(=O)Nc3ccc(Cc4ccc(C)cc4)cc3)cc2)cc1</smiles>

FIGURE 6: The possible interactions between TPU and MWCNT-Ns. The distances among the functional groups are drawn randomly. They give no information about the strength of hydrogen bonding. Two headed arrows and dashed lines show repulsion forces and intermolecular hydrogen bonding, respectively.

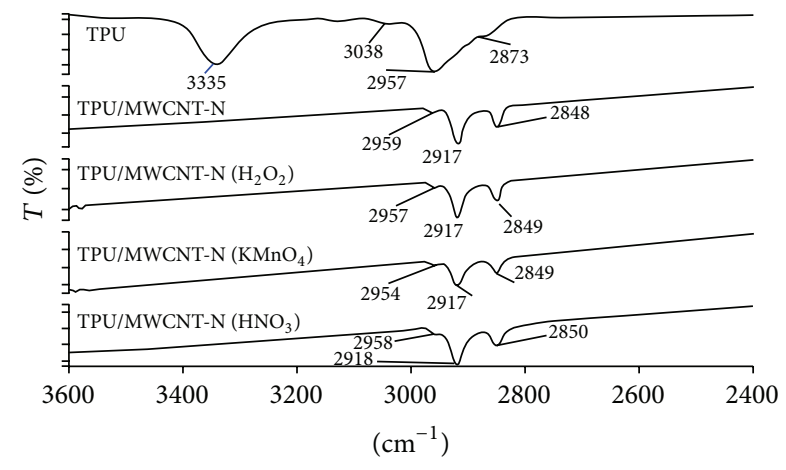

FIGURE 7: FTIR spectra of TPU and the composites between 2400 and $3600 \mathrm{~cm}^{-1}$.

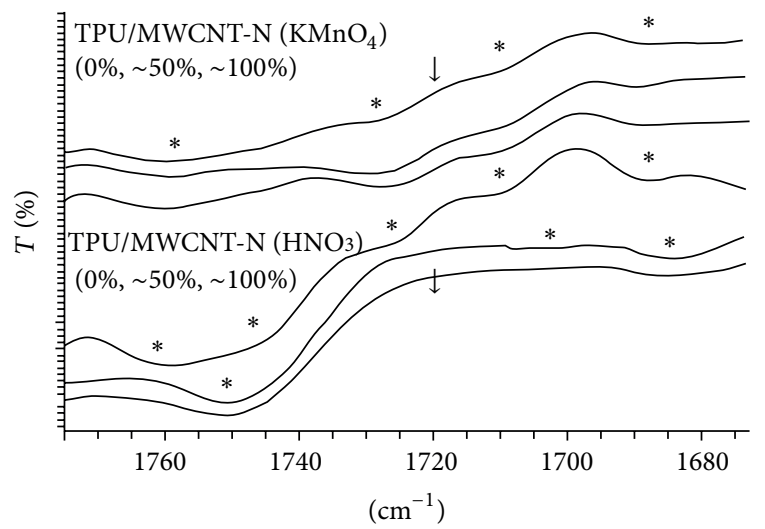

FIgURE 8: Comparison of FTIR spectra of TPU/MWCNT-N $\left(\mathrm{KMnO}_{4}\right)$ and TPU/MWCNT-N $\left(\mathrm{HNO}_{3}\right)$ for various elongations.

shown in Figure 2(c) (upper black layer represents both pure MWCNT network and TPU/MWCNT composite interlayer while lower part is body of TPU specimen). Moreover, the melted polymer apparently encloses MWCNTs from MWCNT network surface in the course of molding at $175^{\circ} \mathrm{C}$, making firm connection between the polymer and layer of

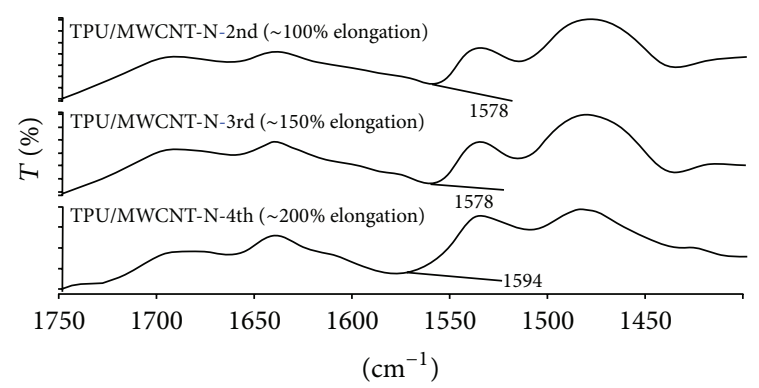

FIGURE 9: FTIR spectra of TPU/MWCNT-N for various elongation.

entangled nanotubes. MWCNT network and TPU filtering membrane can be easily melt welded onto the surface of TPU tensile test specimen (dog-bone shaped) for extension and resistance tests, Figure 2(d). As an example of practical use of TPU/MWCNT composite as a strain sensing element, a strip of TPU/MWCNT-N composite was fixed on the elastic bandage to monitor human elbow flexion.

3.4. Tensile Deformation and Gauge Factor of MWCNTN/PU Composites. The relative electrical resistance change, defined as $\Delta R / R_{0}=\left(R-R_{0}\right) / R_{0}$, where $R_{0}$ is the electrical resistance of the measured sample before the first elongation and $R$ is the resistance while elongating, versus the percentage of mechanical strain is presented in Figure 3. The composite specimens are deformed by the tensile stress with increasing value in each cycle $0.43,0.73,1.07,1.37,1.71$, 2.09 , and $2.47 \mathrm{MPa}$. The increasing stress obviously enhances composite plastic deformation. Consequently, the residual strain increases in the off-load state with increasing number of cycles. The similar residual increase of the resistance is also observed except the resistance of TPU/MWCNT-N $\left(\mathrm{KMnO}_{4}\right)$ composite which is reversible. Thus the nanotube oxidation by $\mathrm{KMnO}_{4}$ has a stabilizing effect on the cyclic resistance change. The testing has shown also the sixteen-fold increase in the resistance for the composite prepared from 


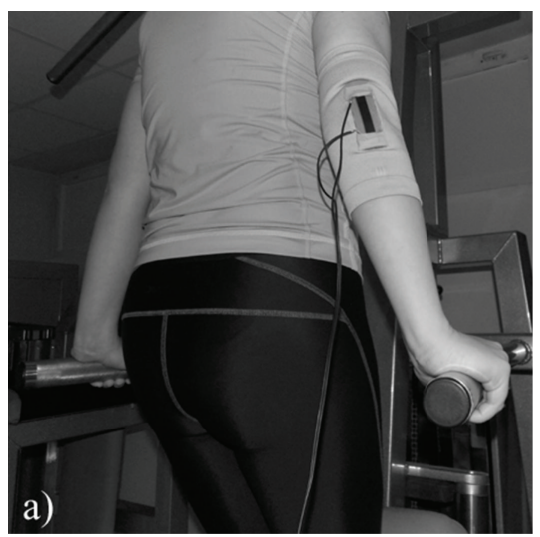

(a)

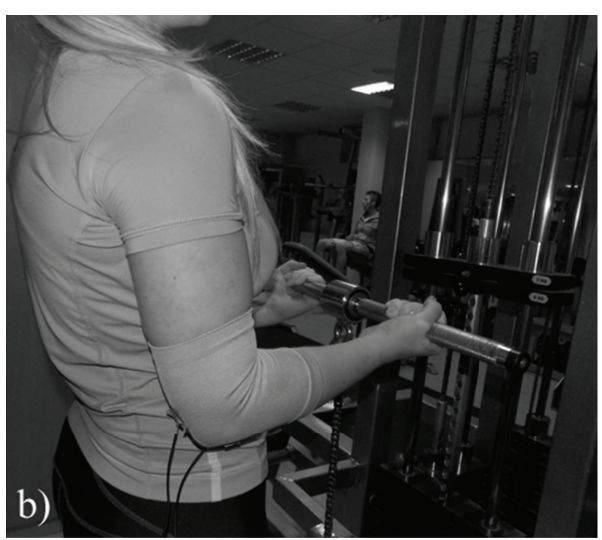

(b)

Figure 10: Measurement of elbow joint flexion during (a) the bodyweight dips and (b) the cable-based arm curls.

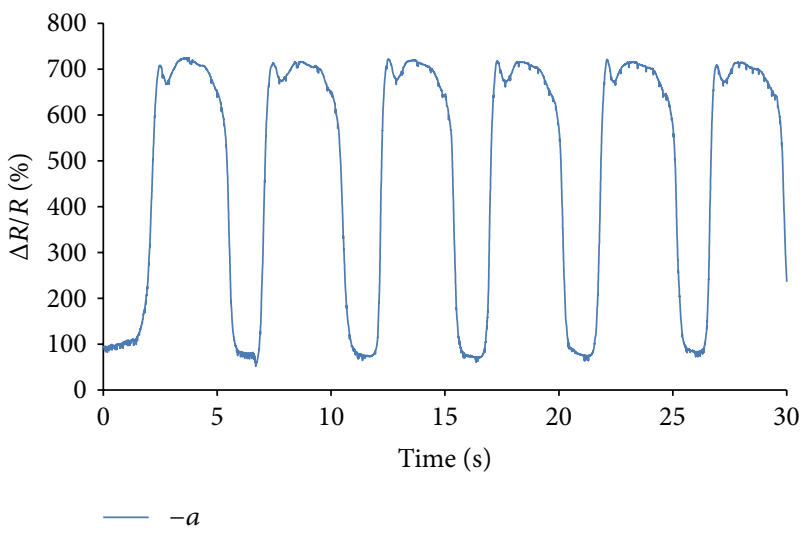

(a)

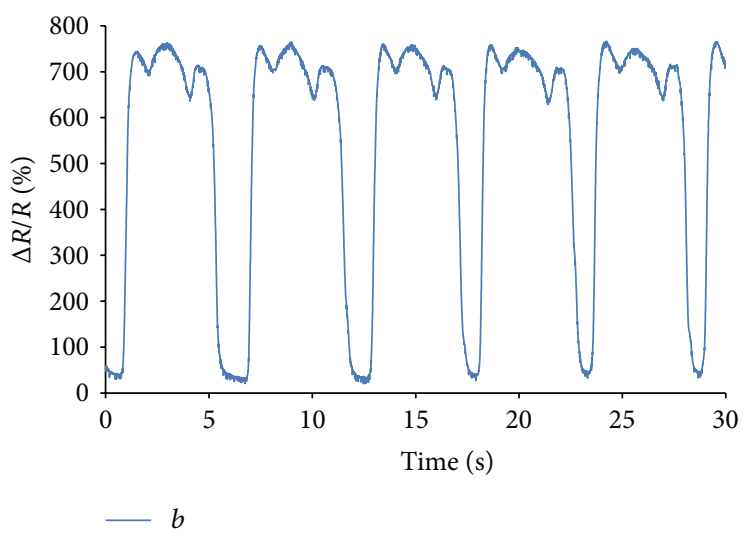

(b)

FIGURE 11: Time-dependent resistance change of TPU/MWNCT-N $\left(\mathrm{KMnO}_{4}\right)$ composite strain sensor during (a) the bodyweight dips and (b) the cable-based arm curls.

$\mathrm{KMnO}_{4}$ oxidized nanotubes in comparison to the composites prepared from the pristine and other oxidized nanotubes.

Figure 4 shows fast resistance change of the strips of composites anchored tightly on one side to bending deformation induced by the tip deflection of free ending. The oxidation treatment evidently causes the increase in the resistance change which is highest for TPU/MWCNT-N $\left(\mathrm{KMnO}_{4}\right)$ composite.

The sensitivity of electrically conductive materials is usually evaluated by a gauge factor GF which is defined as the relative resistance change divided by the applied strain $\varepsilon$, $\mathrm{GF}=\left(\Delta R / R_{0}\right) / \varepsilon[26]$. The gauge factors of the composites given in Figure 5 were calculated by using maximum values of resistance change and strain for each cycle in Figure 3. The gauge factor of CNT thin film was found to be 65 under room temperature for very low strain up to $0.05 \%$ [27]. Wang and coworkers found the Gauge factors of iodine-doped and iodine-undoped carbon nanotubes as 125 and 65, respectively [28].

The calculated gauge factors of our composites increase with the strain up of the maximum measured gauge factor which is for TPU/MWCNT-N, TPU/MWCNT-N $\left(\mathrm{H}_{2} \mathrm{O}_{2}\right)$,
TPU/MWCNT-N $\left(\mathrm{HNO}_{3}\right)$, and TPU/MWCNT-N $\left(\mathrm{KMnO}_{4}\right)$ about $8,10,12$, and 175 for the strains $11,9.1,8.6$ and $10.9 \%$, respectively.

3.5. FTIR Spectra of the Composite Interfaces. As shown above, the type of MWCNTs significantly affects the resulting sensitivity of composites to strain. Rein and coworkers suggested in their study [8] that the source of the large difference in sensitivity between carbon nanotube network types in epoxy resins is the interaction between the polymer and MWCNTs [13]. The difference in the maximum strain values of the investigated composites could arise from the interactions in MWCNT-Ns and/or between TPU and MWCNT-Ns. At first FTIR spectra for the interface of the composites were measured using the MWCNT-Ns as a background.

In the polyester-based polyurethane, the $\mathrm{NH}$ groups as proton donor and the oxygens in carbonyl groups of urethane and ester groups as proton acceptors behave in formation of hydrogen bonding. When MWCNT-Ns have hydroxyl, carboxyl, ketone, and ether groups on their surface, these groups can also play a part in forming hydrogen bonding 
TABLE 2: Some peaks in FTIR spectra of TPU and TPU/MWCNT-Ns composites.

\begin{tabular}{|c|c|c|c|c|c|}
\hline \multirow{2}{*}{ Possible assignments } & \multicolumn{5}{|c|}{ Wavenumber $\left(\mathrm{cm}^{-1}\right)$} \\
\hline & TPU & TPU/MWCNT-N & $\begin{array}{c}\text { TPU/MWCNT-N } \\
\left(\mathrm{H}_{2} \mathrm{O}_{2}\right)\end{array}$ & $\begin{array}{c}\text { TPU/MWCNT-N } \\
\left(\mathrm{HNO}_{3}\right)\end{array}$ & $\begin{array}{c}\text { TPU/MWCNT-N } \\
\left(\mathrm{KMnO}_{4}\right)\end{array}$ \\
\hline $\mathrm{C}=\mathrm{O}$ stretch & - & 1759,1744 & 1748 & 1759,1743 & 1759 \\
\hline Free $\mathrm{C}=\mathrm{O}$ stretch & 1728 & 1726 & 1715 & 1723 & 1725 \\
\hline $\begin{array}{l}\mathrm{C}=\mathrm{O} \text { stretch for hydrogen } \\
\text { bonded urethane group }\end{array}$ & 1706 & 1710,1690 & 1700,1683 & 1708,1689 & 1707,1688 \\
\hline $\begin{array}{l}\mathrm{C}=\mathrm{O} \text { stretch for hydrogen } \\
\text { bonded ester group }\end{array}$ & 1662 & 1665 & 1652 & 1664 & 1662 \\
\hline $\mathrm{NH}$ bending $+\mathrm{C}=\mathrm{C}$ stretch & 1597 & 1574 & 1573 & 1574 & 1577 \\
\hline $\begin{array}{l}\mathrm{C}-\mathrm{N} \text { stretch }+\mathrm{N}-\mathrm{H} \\
\text { bending }\end{array}$ & 1531 & 1535 & 1538 & 1539 & 1539 \\
\hline
\end{tabular}

between the polymer and MWCNT-Ns. Possible interactions between TPU and MWCNT-Ns are shown in Figure 6 by considering FTIR and XPS data of MWCNT-Ns.

Some changes observed in FTIR spectra of TPU/MWCNT composites compared with that of pure TPU are given in Figure 7 and Table 2. The broad $-\mathrm{NH}$ absorption between 3230 and $3430 \mathrm{~cm}^{-1}$ has been removed in TPU/MWCNT-N composites which could show that all $\mathrm{NH}$ groups are hydrogen bonded with some groups on the MWNTs. The $\mathrm{CH}$ bands $\left(\mathrm{CH}_{2} / \mathrm{CH}_{3}\right)$ seen at 2957 and $2873 \mathrm{~cm}^{-1}$ in TPU are divided in the composites into three parts such as around 2960,2917 , and $2850 \mathrm{~cm}^{-1}$ due to the interaction with MWCNT-Ns.

The observed changes in three different carbonyl peaks of TPU can be seen in Table 2. When the ones at 1728 and $1706 \mathrm{~cm}^{-1}$ split into two or three parts in the composites due to some interactions shown in Figure 6, some of three carbonyl peaks remained unchanged or shifted to higher/lower wavelengths for different composites. The more shifting to higher wavelength for all carbonyl peaks was observed for TPU/MWCNT-N $\left(\mathrm{H}_{2} \mathrm{O}_{2}\right)$ due to probable hydrogen bonding between the $\mathrm{C}=\mathrm{O}$ groups on TPU and $-\mathrm{OH}$ groups on the MWCNT-N.

3.6. FTIR Spectra of the Composite Interfaces Subjected to Strain. FTIR spectra related to the composite interfaces reveal some differences among the amounts of functional groups on the MWCNT-Ns. Hence FTIR spectra of TPU and the interfaces of TPU composites subjected to 7 various strains were examined. The values of strain could not be calculated properly due to the pressure of the ATR tip on the samples. Important differences among them are given in Figures 8 and 9.

Some peaks in the $\mathrm{C}=\mathrm{O}$ stretch region of TPU/MWCNT$\mathrm{N}\left(\mathrm{HNO}_{3}\right)$ which could interact with the $-\mathrm{OH} /-\mathrm{COOH}$ groups on the MWCNT-N disappeared or shifted to lower wavenumber in the 2 nd and 3 rd elongations as follows from Figure 8, owing to new interactions of carbonyl groups on TPU with the functional groups on MWCNT-N $\left(\mathrm{HNO}_{3}\right)$. On the other hand, the peaks in the $\mathrm{C}=\mathrm{O}$ stretch region of TPU/MWCNT-N $\left(\mathrm{KMnO}_{4}\right)$ remained unchanged. Figure 9 shows that hydrogen bond between $\mathrm{NH}$ group in TPU and $\mathrm{C}=\mathrm{O} /-\mathrm{OH} /-\mathrm{COOH}$ group in the pristine MWCNT$\mathrm{N}$, schematically illustrated in Figure 6, was damaged in the 4 th elongation. Such a situation was not observed for other TPU composites. The reason seems to be that there are less oxygenated groups on the pristine MWCNT-N in comparison with oxidized nanotube networks (Figure 1). The disappeared/changed interactions at interfaces of the composites with strain may influence the stress transfer between TPU and MWCNT networks. Hence, the observation may also give information about why TPU/MWCNT-N $\left(\mathrm{HNO}_{3}\right)$ and TPU/MWCNT-N have lower resistance change with strain than TPU/MWCNT-N $\left(\mathrm{KMnO}_{4}\right)$ when the interactions between MWCNTs may be affected due to the difference in the amount of functional groups on their surfaces.

3.7. Practical Use of TPU/MWCNT-N $\left(\mathrm{KMnO}_{4}\right)$ Composite. The possible practical use of TPU/MWCNT-N $\left(\mathrm{KMnO}_{4}\right)$ composite is demonstrated by monitoring of elbow joint flexion during two different physical exercises, namely, bodyweight dips and cable-based arm curls (Figure 10). The strip of the composite was adhered to an elastic bandage $(83 \%$ of polyamide and $17 \%$ of elastolan) by means of dimethyl formamide/methyl isobutyl ketone (1:3) solution. The electrical contacts were fixed to the strip by a silver-colloid electroconductive paint Dotite D-550 (SPI Supplies), and the resistance was measured lengthwise by the two-point technique using multimeter Sefram 7338.

Figures 11(a) and 11(b) show waveforms of the test performed by the volunteer with the bandage. The data are reversible and sensitive so that the composite can be used, for example, for the similar measurements in orthopedics and rehabilitation.

\section{Conclusions}

Multi-walled carbon nanotubes were used in their pure and oxidized forms to prepare entangled networks as a part of TPU/MWCNT-N composites. The response of composites to deformation was measured by their electrical resistance change in the course of extension/relaxation cycles and bending. The results show that the composite with $\mathrm{KMnO}_{4}$ oxidized nanotube network has an enhanced electrical resistance 
with loading in comparison with the network prepared from the pristine and other oxidized nanotubes. The evaluated sensitivity of TPU/MWCNT-N $\left(\mathrm{KMnO}_{4}\right)$ composite in terms of the gauge factor increases with strain from values around 5 at the start of deformation to 175 at the strain $11 \%$. This is a substantial increase, which ranks the composite among the materials with the highest electromechanical sensitivity.

The distinct resistance and sensitivity increase with elongation and bending may come probably from the different porous structure of MWCNT $\left(\mathrm{KMnO}_{4}\right)$ network, the interaction between TPU and MWCNT-N $\left(\mathrm{KMnO}_{4}\right)$ and the increased number of carboxyl groups detached on MWCNT$\mathrm{N}\left(\mathrm{KMnO}_{4}\right)$ surface. The short oxidized nanotubes, forming densely packed network with small pores, probably lose mechanical contacts with other ones more easily in the course of composite elongation than the longer pristine nanotubes. The unchanged interactions between TPU and MWCNT-N $\left(\mathrm{KMnO}_{4}\right)$ at the composite interface during the elongation, discussed in details in Section 3.6, may ensure stress transfer from the elastic TPU to the network and its extensive rearrangement and loss of nanotube contacts. Similarly, the increased presence of carboxyl groups on the surface of MWCNT-N $\left(\mathrm{KMnO}_{4}\right)$ in comparison with other investigated networks (Figure 1) enhances interaction of nanotubes via two interaction points (carbonyl and hydroxyl groups). Thus during elongation more chemical links between nanotubes fade which results also in loss of conductive points, and consequently, the resistance rise.

\section{Conflict of Interests}

Petr Slobodian declares that there is no conflict of interests.

\section{Acknowledgments}

The work was supported by the internal Grant of TBU in Zlin No. IGA/FT/2013/018 funded from the resources of Specific University Research and Operational Program of Research and Development for Innovations cofunded by the European Regional Development Fund (ERDF), the national budget of Czech Republic within the framework of the Centre of Polymer Systems project (reg. no. CZ.1.05/2.1.00/03.0111). This paper was also written with the support of the Operational Programme "Education for Competitiveness" co-funded by the European Social Fund (ESF) and the national budget of the Czech Republic, within the "Advanced Theoretical and Experimental Studies of Polymer Systems" project (reg. no. CZ.1.07/2.3.00/20.0104). The Fund of Institute of Hydrodynamics, no. of project AV0Z20600510, is also acknowledged for support.

\section{References}

[1] A. K. Barick and D. K. Tripathy, "Preparation, characterization and properties of acid functionalized multi-walled carbon nanotube reinforced thermoplastic polyurethane nanocomposites," Materials Science and Engineering B, vol. 176, no. 18, pp. 14351447, 2011.
[2] A. K. Barick and D. K. Tripathy, "Effect of nanofiber on material properties of vapor-grown carbon nanofiber reinforced thermoplastic polyurethane (TPU/CNF) nanocomposites prepared by melt compounding," Composites Part A, vol. 41, no. 10, pp. 1471-1482, 2010.

[3] Q. Fan, Z. Qin, T. Villmow et al., "Vapor sensing properties of thermoplastic polyurethane multifilament covered with carbon nanotube networks," Sensors and Actuators B, vol. 156, no. 1, pp. 63-70, 2011.

[4] P. Slobodian, P. Riha, and P. Saha, "A highly-deformable composite composed of an entangled network of electricallyconductive carbon-nanotubes embedded in elastic polyurethane," Carbon, vol. 50, no. 10, pp. 3446-3453, 2012.

[5] A. M. F. Lima, V. G. de Castro, R. S. Borges, and G. G. Silva, "Electrical conductivity and thermal properties of functionalized carbon nanotubes/polyurethane composites," Polímeros, vol. 22, pp. 117-124, 2012.

[6] D. M. Crawford, R. G. Bass, and T. W. Haas, "Strain effects on thermal transitions and mechanical properties of thermoplastic polyurethane elastomers," Thermochimica Acta, vol. 323, no. 1-2, pp. 53-63, 1998.

[7] R. Olejnik, P. Slobodian, P. Riha, and P. Saha, "An electricallyconductive and organic solvent vapors detecting composite composed of an entangled network of carbon nanotubes embedded in polystyrene," Journal of Nanomaterials, vol. 2012, Article ID 365062, 7 pages, 2012.

[8] M. D. Rein, O. Breuer, and H. D. Wagner, "Sensors and sensitivity: carbon nanotube buckypaper films as strain sensing devices," Composites Science and Technology, vol. 71, no. 3, pp. 373-381, 2011.

[9] I. Kang, M. J. Schulz, J. H. Kim, V. Shanov, and D. Shi, "A carbon nanotube strain sensor for structural health monitoring," Smart Materials and Structures, vol. 15, no. 3, pp. 737-748, 2006.

[10] Q. Jiang, Y. Li, J. Xie, J. Sun, D. Hui, and Y. Qiu, "Plasma functionalization of bucky paper and its composite with phenylethynyl-terminated polyimide," Composites Part B, vol. 45, pp. 1275-1281, 2013.

[11] P. Pötschke, N. P. Zschoerper, B. P. Moller, and U. Vohrer, "Plasma functionalization of multiwalled carbon nanotube bucky papers and the effect on properties of melt-mixed composites with polycarbonate," Macromolecular Rapid Communications, vol. 30, no. 21, pp. 1828-1833, 2009.

[12] Z. Špitalský, C. A. Krontiras, S. N. Georga, and C. Galiotis, "Effect of oxidation treatment of multiwalled carbon nanotubes on the mechanical and electrical properties of their epoxy composites," Composites Part A, vol. 40, no. 6-7, pp. 778-783, 2009.

[13] M. D. Rein, H. Bar, O. Breuer, R. Yoseph, and H. D. Wagner, "Electromechanical properties of carbon nanotube buckypapers," in Proceedings of the 17th International Conference on Composite Materials (ICCM-17 '09), Edinburgh, UK, July 2009.

[14] P. Slobodian, P. Riha, A. Lengalova, P. Svoboda, and P. Saha, "Multi-wall carbon nanotube networks as potential resistive gas sensors for organic vapor detection," Carbon, vol. 49, no. 7, pp. 2499-2507, 2011.

[15] P. A. Jansson, Ed., Deconvolution of Spectra and Images, Academic Press, San Diego, Calif, USA, 1997.

[16] F. A. Abuilaiwi, T. Laoui, M. Al-Harthi, and M. A. Atieh, "Modification and functionalization of multiwalled carbon nanotube (MWCNT) via fischer esterification," Arabian Journal for Science and Engineering, vol. 35, no. 1, pp. 37-48, 2010. 
[17] J. Zhang, H. Zou, Q. Qing et al., "Effect of chemical oxidation on the structure of single-walled carbon nanotubes," Journal of Physical Chemistry B, vol. 107, no. 16, pp. 3712-3718, 2003.

[18] P. E. Fanning and M. A. Vannice, "A DRIFTS study of the formation of surface groups on carbon by oxidation," Carbon, vol. 31, no. 5, pp. 721-730, 1993.

[19] T. G. Ros, A. J. Van Dillen, J. W. Geus, and D. C. Koningsberger, "Surface oxidation of carbon nanofibres," Chemistry, vol. 8, pp. 1151-1162, 2002.

[20] C. Moreno-Castilla, M. V. López-Ramón, and F. CarrascoMarín, "Changes in surface chemistry of activated carbons by wet oxidation," Carbon, vol. 38, no. 14, pp. 1995-2001, 2000.

[21] U. J. Kim, C. A. Furtado, X. Liu, G. Chen, and P. C. Eklund, "Raman and IR spectroscopy of chemically processed singlewalled carbon nanotubes," Journal of the American Chemical Society, vol. 127, no. 44, pp. 15437-15445, 2005.

[22] Y. Liang, H. Zhang, B. Yi, Z. Zhang, and Z. Tan, "Preparation and characterization of multi-walled carbon nanotubes supported PtRu catalysts for proton exchange membrane fuel cells," Carbon, vol. 43, no. 15, pp. 3144-3152, 2005.

[23] K. A. Wepasnick, B. A. Smith, K. E. Schrote, H. K. Wilson, S. R. Diegelmann, and D. H. Fairbrother, "Surface and structural characterization of multi-walled carbon nanotubes following different oxidative treatments," Carbon, vol. 49, no. 1, pp. 24-36, 2011.

[24] J. Chen, Q. Chen, and Q. Ma, "Influence of surface functionalization via chemical oxidation on the properties of carbon nanotubes," Journal of Colloid and Interface Science, vol. 370, no. 1, pp. 32-38, 2012.

[25] H.-T. Fang, C.-G. Liu, C. Liu, F. Li, M. Liu, and H.-M. Cheng, "Purification of single-wall carbon nanotubes by electrochemical oxidation," Chemistry of Materials, vol. 16, no. 26, pp. 57445750, 2004.

[26] P. Slobodian, R. Olejnik, P. Riha, and P. Saha, "Effect of functionalized nanotubes with $\mathrm{HNO}_{3}$ on electrical sensory properties of carbon nanotubes/polyurethane composite under elongation," in Mathematical Methods and Techniques in Engineering and Environmental Science, M. Demiralp, Z. Bojkovic, and A. Repanovici, Eds., pp. 312-316, WSEAS Press, 2011.

[27] T. W. Tombler, C. Zhou, L. Alexseyev et al., "Reversible electromechanical characteristics of carbon nanotubes under localprobe manipulation," Nature, vol. 405, no. 6788, pp. 769-772, 2000.

[28] W.-L. Wang, K.-J. Liao, Y. Li, and Y.-T. Wang, "Piezoresistive effect of doped carbon nanotube/cellulose films," Chinese Physics Letters, vol. 20, no. 9, pp. 1544-1547, 2003. 

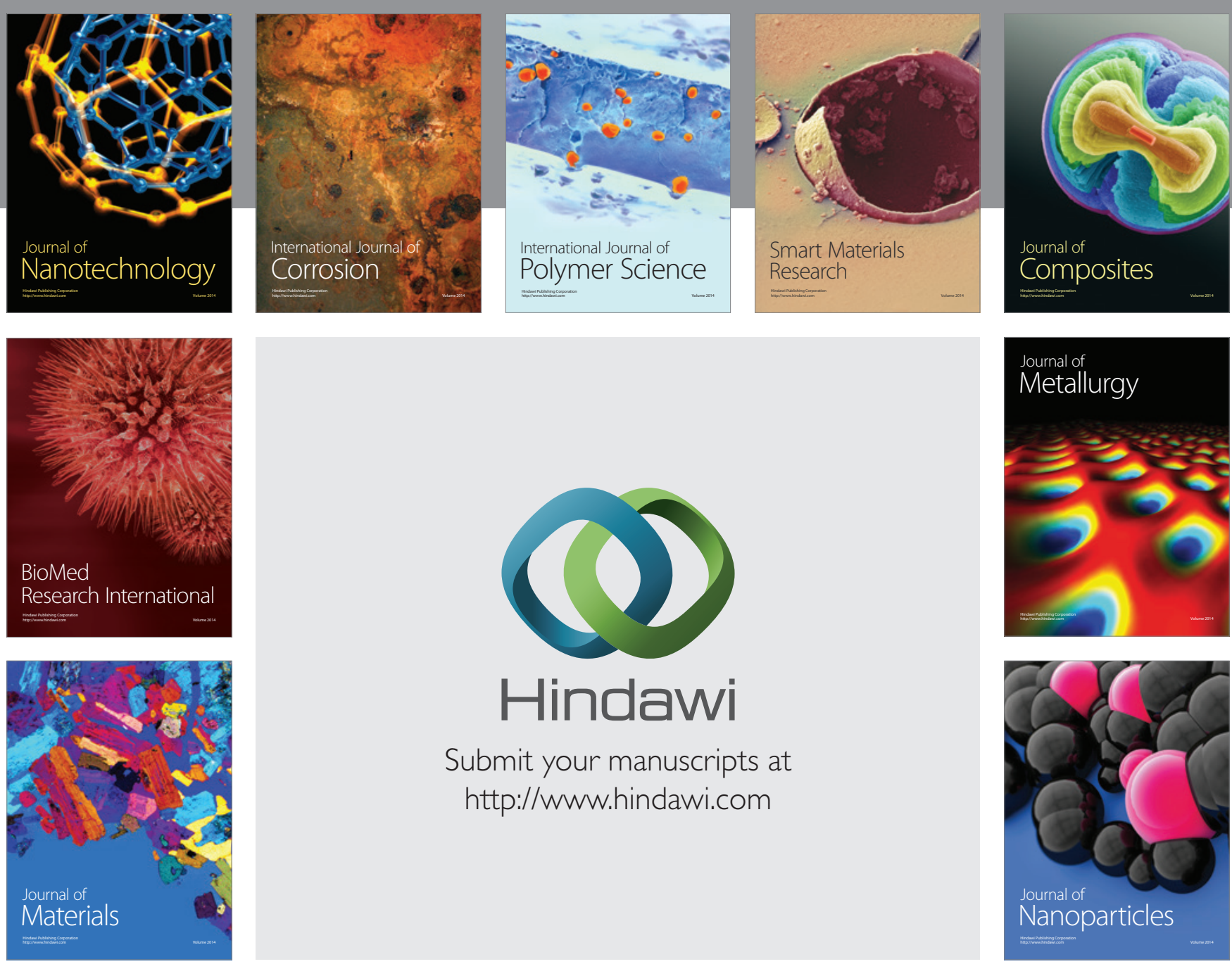

Submit your manuscripts at http://www.hindawi.com
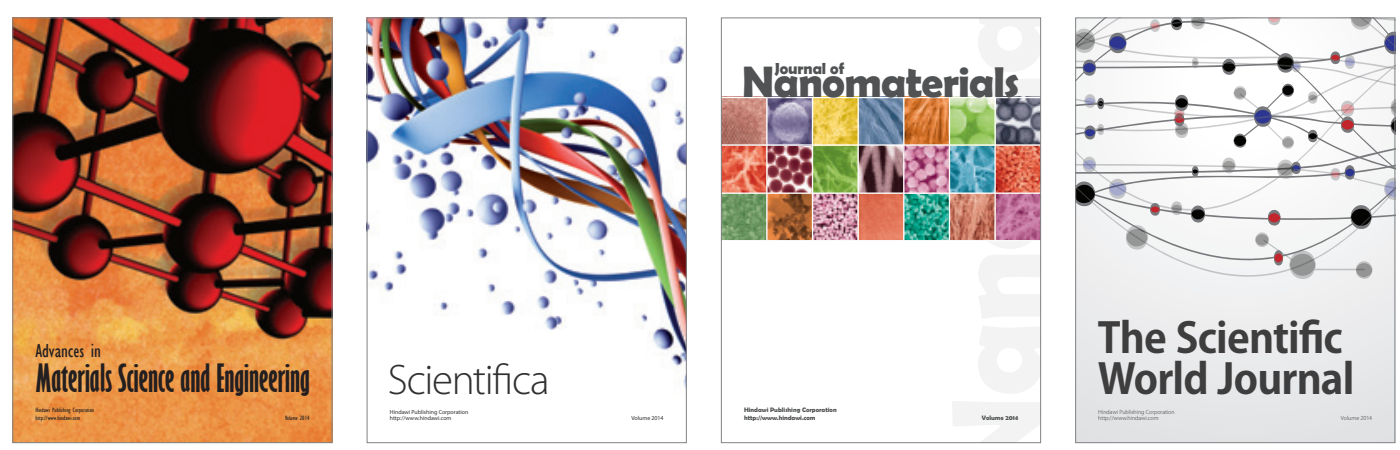

\section{The Scientific World Journal}
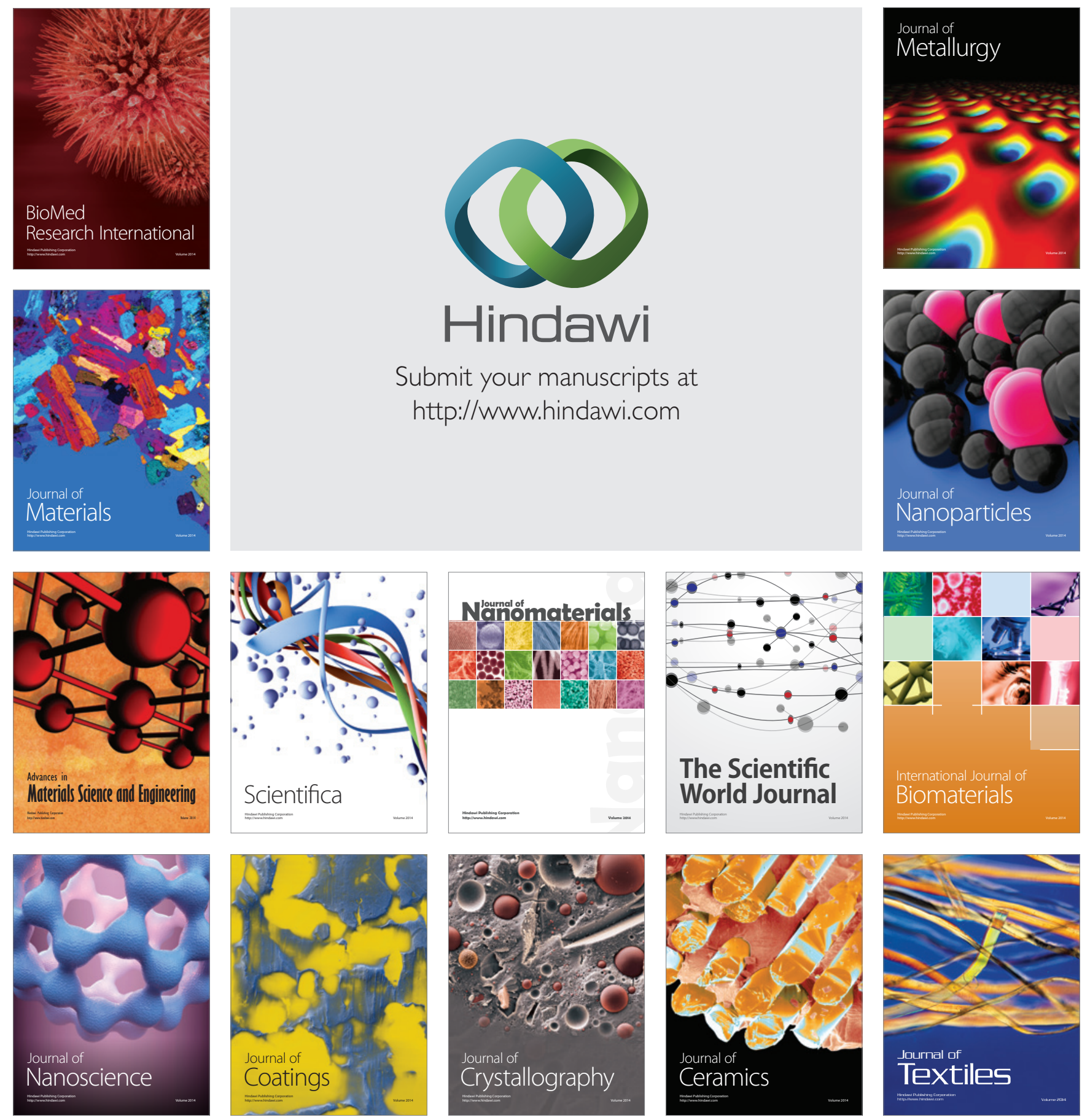